\title{
Outage probability of decode-and-forward cooperative diversity over correlated shadowed fading channels
}

\author{
Liang Han ${ }^{1,2 a)}$, Ying Liu ${ }^{2)}$, Shihai Shao ${ }^{2)}$, and Youxi Tang ${ }^{2)}$ \\ ${ }^{1}$ College of Electronic and Communication Engineering,
}

Tianjin Normal University, Tianjin 300387, China

${ }^{2}$ National Key Lab of Science and Technology on Communications, University of Electronic Science and Technology of China, Chengdu 611731, China a)hanliang@uestc.edu.cn

Abstract: In this letter, we investigate the effect of correlated lognormal shadowing on the outage probability of decode-and-forward (DF) cooperative diversity systems. Since there is no closed-form expression for the outage probability, we present a numerical approach to evaluate the outage probability by using a decorrelating transformation and Gaussian-Hermite integration technique. The numerical results verify that our proposed approach is accurate, and show that the correlation coefficients and standard deviations of shadowing have a significant impact on the outage performance.

Keywords: decode-and-forward, outage probability, correlated shadowing

Classification: Wireless Communication Technologies

\section{References}

[1] J. N. Laneman, D. N. C. Tse, and G. W. Wornell, "Cooperative diversity in wireless networks: efficient protocols and outage behavior," IEEE Trans. Inf. Theory, vol. 50, no. 12, pp. 3062-3080, Dec. 2004.

[2] G. Kramer, M. Gastpar, and P. Gupta, "Cooperative strategies and capacity theorems for relay networks," IEEE Trans. Inf. Theory, vol. 51, no. 9, pp. 3037-3063, Sept. 2005.

[3] P. Agrawal and N. Patwari, "Correlated link shadow fading in multihop wireless networks," IEEE Trans. Wireless Commun., vol. 8, no. 8, pp. 4024-4036, Aug. 2009.

[4] S. S. Szyszkowicz, H. Yanikomeroglu, and J. S. Thompson, "On the feasibility of wireless shadowing correlation models," IEEE Trans. Veh. Technol., vol. 59, no. 9, pp. 4222-4236, Nov. 2010.

[5] M. Safari and M. Uysal, "Cooperative diversity over log-normal fading channels: performance analysis and optimization," IEEE Trans. Wireless Commun., vol. 7, no. 5, pp. 1963-1972, May 2008.

[6] M. D. Renzo, F. Graziosi, and F. Santucci, "A comprehensive framework for performance analysis of cooperative multi-hop wireless systems 
over log-normal fading channels," IEEE Trans. Commun., vol. 58, no. 2, pp. 531-544, Feb. 2010.

[7] G. Pan and Q. Feng, "Performance analysis of DF relaying multi-hop systems over log-normal fading channels," Int. J. Electron. Commun., vol. 67, no. 6, pp. 457-462, June 2013.

[8] V. K. Sakarellos, D. Skraparlis, and A. D. Panagopoulos, "Cooperative radio communications over correlated shadowing: outage analysis," Future Network \&6 Mobile Summit 2012, Berlin, Germany, pp. 1-8, July 2012.

[9] M. D. Renzo, F. Graziosi, and F. Santucci, "A unified framework for performance analysis of CSI-assisted cooperative communications over fading channels," IEEE Trans. Commun., vol. 57, no. 9, pp. 2551-2557, Sept. 2009.

[10] M. D. Renzo, F. Graziosi, and F. Santucci, "A comprehensive framework for performance analysis of dual-hop cooperative wireless systems with fixed-gain relays over generalized fading channels," IEEE Trans. Wireless Commun., vol. 8, no. 10, pp. 5060-5074, Oct. 2009.

[11] H. Yu, I. Lee, and G. L. Stüber, "General decode-and-forward cooperative relaying with co-channel interference in shadowed Nakagami fading channels," IEEE Trans. Wireless Commun., vol. 11, no. 12, pp. 43184327, Dec. 2012.

[12] Y. Tsai and L. Lin, "Optimal power allocation for decode-and-forward cooperative diversity under an outage performance constraint," IEEE Commun. Lett., vol. 14, no. 10, pp. 945-947, Oct. 2010.

[13] M. K. Simon and M.-S. Alouini, Digital Communication over Fading Channels: A Unified Approach to Performance Analysis, Wiley, New York, 2000.

\section{Introduction}

Cooperative diversity techniques have been regarded as an effective way to improve the transmission reliability and extend the network coverage $[1,2]$. Although the performance analysis for various cooperative diversity schemes, such as amplify-and-forward (AF) and decode-and-forward (DF) has extensively been studied, most of the current works were mainly limited to the cases that only considered the small-scale fading, e.g., Rayleigh, Rician or Nakagami fading, and few works have worked on the cooperative diversity considering the large-scale fading.

The large-scale fading, also known as shadowing, is caused by obstacles, e.g., buildings and hills, between the transmitter and receiver, and often modeled by a lognormal distribution. For the cooperative diversity systems, the source, relay and destination are distributed in different locations, and thus the effects of shadowing between any two of them are more likely to be different and correlated, which has also been demonstrated by empirical measurements $[3,4]$. In [5], the authors investigated the bit error rate of single-relay AF relaying over lognormal channels. The performance analysis of multi-hop AF and DF cooperative relaying systems over lognormal channels were investigated in [6] and [7], respectively. In [8], the outage per- 
formance of DF relaying over correlated lognormal channels was investigated. However, these papers were limited to the channels of pure lognormal distribution, and they have not considered the composite fading channels which includes small and large scale fadings (i.e., shadowed fading channels). In [9] and [10], the authors proposed a comprehensive framework for performance analysis of cooperative AF relay systems over generalized fading channels. In [11], the selection DF multi-branch multi-hop cooperative relaying systems were investigated on non-identical shadowed Nakagami- $m$ fading channels in the presence of non-identical co-channel interferers. However, these papers only considered independent shadowed fading channels.

In this letter, we study the outage probability of DF cooperative diversity systems that use selection combining at the destination, considering the correlated lognormal shadowing and independent Rayleigh fading. Since there is no closed-form for the outage probability, Gaussian-Hermite integration technique is applied to evaluate the outage probability, and the accuracy of our expression is also demonstrated by comparing with Monte-Carlo simulation.

\section{Channel model}

Consider a three-node relay channel, where a source communicates with a destination through a single relay. We assume that the channels between these nodes consist of small-scale Rayleigh fading and large-scale lognormal shadowing. Denote the signal-to-noise ratio (SNR) of the source-destination, source-relay, and relay-destination links as $\gamma_{1}, \gamma_{2}$ and $\gamma_{3}$, respectively. Due to the combination of small-scale fading and large-scale shadowing, the SNR $\gamma_{i}, i=1,2,3$, follows a composite exponential lognormal distribution [13] and thus $\gamma=\left(\gamma_{1}, \gamma_{2}, \gamma_{3}\right)^{T}$ follows a multivariate joint composite exponential lognormal distribution. The probability density function (PDF) of $\gamma$ is given by

$$
f_{\boldsymbol{\gamma}}(\boldsymbol{x})=\int_{0}^{\infty} \int_{0}^{\infty} \int_{0}^{\infty} f_{\gamma \mid \boldsymbol{\xi}}(\boldsymbol{x} \mid \boldsymbol{y}) f_{\boldsymbol{\xi}}(\boldsymbol{y}) d \boldsymbol{y}
$$

where $f_{\gamma \mid \boldsymbol{\xi}}(\boldsymbol{x} \mid \boldsymbol{y})$ denotes the joint conditional PDF of the squared envelope vector of small-scale fading, conditioned on the local mean SNR vector $\boldsymbol{\xi}=\left(\xi_{1}, \xi_{2}, \xi_{3}\right), f_{\boldsymbol{\xi}}(\boldsymbol{y})$ denotes the joint PDF of the local mean SNR vector, and the multivariate integrals are evaluated over the elements of vector $\boldsymbol{y}=\left(y_{1}, y_{2}, y_{3}\right)^{T}$. Since all nodes are separately deployed far away from each other, the small-scale Rayleigh fading is assumed to be independent and thus the joint conditional PDF in (1) is given by

$$
f_{\boldsymbol{\gamma} \mid \boldsymbol{\xi}}(\boldsymbol{x} \mid \boldsymbol{y})=\prod_{i=1}^{3}\left[\frac{1}{y_{i}} \exp \left(-\frac{x_{i}}{y_{i}}\right)\right] .
$$

However, measurements in practical mobile communication environments have shown that the lognormal shadowings are usually correlated $[3,4]$. For correlated lognormal shadowing, the local mean SNR vector $\boldsymbol{\xi}$ follows a mul- 
tivariate lognormal distribution, and its joint $\mathrm{PDF}$ is given by

$$
\begin{aligned}
f_{\boldsymbol{\xi}}(\boldsymbol{y}) & =\frac{\eta^{3}}{(2 \pi)^{3 / 2}|\mathbf{C}|^{1 / 2} \prod_{i=1}^{3} y_{i}} \\
& \cdot \exp \left(-\frac{1}{2}\left(10 \log _{10} \boldsymbol{y}-\boldsymbol{\mu}\right)^{T} \mathbf{C}^{-1}\left(10 \log _{10} \boldsymbol{y}-\boldsymbol{\mu}\right)\right)
\end{aligned}
$$

where $\eta=10 / \ln 10$, the vector $\boldsymbol{\mu}=\left(\mu_{1}, \mu_{2}, \mu_{3}\right)$ is the mean SNR in $\mathrm{dB}$ with only path loss, $|\cdot|$ denotes the determinant, and $\mathbf{C}$ is a symmetric $3 \times 3$ shadowing covariance matrix, which is given as

$$
\mathbf{C}=\left[\begin{array}{ccc}
\sigma_{1}^{2} & \rho_{12} \sigma_{1} \sigma_{2} & \rho_{13} \sigma_{1} \sigma_{3} \\
\rho_{12} \sigma_{1} \sigma_{2} & \sigma_{2}^{2} & \rho_{23} \sigma_{2} \sigma_{3} \\
\rho_{13} \sigma_{1} \sigma_{3} & \rho_{23} \sigma_{2} \sigma_{3} & \sigma_{3}^{2}
\end{array}\right]
$$

where $\sigma_{i}$ is the standard deviation of $10 \log _{10} \xi_{i}$, and $\rho_{i j}$ is the correlation coefficient between $10 \log _{10} \xi_{i}$ and $10 \log _{10} \xi_{j}$.

\section{Outage probability analysis}

In this section, we derive the outage probability for the DF cooperative diversity systems over correlated shadowed fading channels. The DF protocol is carried out in two phases. In the first phase, the source transmits a signal to the relay and the destination. If the received SNR at the relay is above the specified threshold $\Gamma$, then the relay is assumed to be able to decode the source message successfully and forward it to the destination in the second phase; otherwise, the relay keeps silent. Instead of using maximal ratio combining (MRC), which requires the channel state information (CSI), selection combining (SC) is adopted at the destination [12]. Since the source node transmits the information to the destination via the source-destination or source-relay-destination links, we define two events: $\mathbf{A}=\left\{\gamma_{1}>\Gamma\right\}$ and $\mathrm{B}=\left\{\gamma_{2}>\Gamma \cap \gamma_{3}>\Gamma\right\}$. Then $\mathrm{A} \cup \mathrm{B}$ is the event that the source and destination can communicate, and the outage probability is given as

$$
P_{\text {out }}=1-\operatorname{Pr}(\mathrm{A} \cup \mathrm{B})=1-[\operatorname{Pr}(\mathrm{A})+\operatorname{Pr}(\mathrm{B})-\operatorname{Pr}(\mathrm{A} \cap \mathrm{B})] .
$$

There is no closed-form expression for the outage probability when the channel follows a composite exponential lognormal distribution, so we use Gaussian-Hermite integration to evaluate the outage probability [13]. In [13], the probability for $\operatorname{Pr}(\mathrm{A})$ has been derived as

$$
\operatorname{Pr}(\mathrm{A}) \approx \frac{1}{\sqrt{\pi}} \sum_{n=1}^{N_{p}} H_{n} \exp \left[-\Gamma 10^{-\left(\sqrt{2} \sigma_{1} \theta_{n}+\mu_{1}\right) / 10}\right],
$$

where $\theta_{n}$ and $H_{n}$ are respectively the base points and weight factors of an $N_{p}$-order Hermite polynomial. Therefore, we only need to calculate $\operatorname{Pr}(\mathrm{B})$ and $\operatorname{Pr}(\mathrm{A} \cap \mathrm{B})$ to obtain the outage probability.

In the following, we introduce a new vector $\boldsymbol{z}=\left(z_{1}, z_{2}, z_{3}\right)^{T}$, where $z_{i}=$ $\left(10 \log _{10} y_{i}-\mu_{i}\right) / \sqrt{2} \sigma_{i}$. Consequently, $y_{i}$ can be expressed as a function of 
$z_{i}$ as $y_{i}=g\left(z_{i}\right)=10^{\left(\sqrt{2} \sigma_{i} z_{i}+\mu_{i}\right) / 10}$. Then, we have

$$
\begin{aligned}
& \operatorname{Pr}(\mathrm{A} \cap \mathrm{B})=\int_{\Gamma}^{\infty} \int_{\Gamma}^{\infty} \int_{\Gamma}^{\infty} f_{\boldsymbol{\gamma}}(\boldsymbol{x}) d \boldsymbol{x} \\
& =\int_{\Gamma}^{\infty} \int_{\Gamma}^{\infty} \int_{\Gamma}^{\infty} \int_{0}^{\infty} \int_{0}^{\infty} \int_{0}^{\infty} \prod_{i=1}^{3}\left[\frac{1}{y_{i}} \exp \left(-\frac{x_{i}}{y_{i}}\right)\right] f_{\boldsymbol{\xi}}(\boldsymbol{y}) d \boldsymbol{y} d \boldsymbol{x} \\
& =\int_{0}^{\infty} \int_{0}^{\infty} \int_{0}^{\infty} \prod_{i=1}^{3} \exp \left(-\frac{\Gamma}{y_{i}}\right) f_{\boldsymbol{\xi}}(\boldsymbol{y}) d \boldsymbol{y} \\
& =\frac{1}{(\sqrt{\pi})^{3}|\mathbf{R}|^{\frac{1}{2}}} \int_{-\infty}^{\infty} \int_{-\infty}^{\infty} \int_{-\infty}^{\infty} \exp \left[\sum_{i=1}^{3} \frac{-\Gamma}{g\left(z_{i}\right)}\right] \exp \left(-\boldsymbol{z}^{T} \mathbf{R}^{-1} \boldsymbol{z}\right) d \boldsymbol{z} .
\end{aligned}
$$

where $\mathbf{R}$ is the normalized form of $\mathbf{C}$, given as

$$
\mathbf{R}=\left[\begin{array}{ccc}
1 & \rho_{12} & \rho_{13} \\
\rho_{12} & 1 & \rho_{23} \\
\rho_{13} & \rho_{23} & 1
\end{array}\right]
$$

We assume that $\mathbf{D}$ is the square root of the normalized correlation matrix $\mathbf{R}$, i.e., $\mathbf{R}=\mathbf{D D}^{T}$. To obtain $\mathbf{D}$, let the eigendecomposition of $\mathbf{R}$ be $\mathbf{R}=$ $\mathbf{U} \boldsymbol{\Lambda} \mathbf{U}^{T}$, where $\mathbf{U}$ is the unitary matrix whose columns are the eigenvectors and $\boldsymbol{\Lambda}$ is the diagonal matrix whose diagonal elements are the corresponding eigenvalues of $\mathbf{R}$. Then we have $\mathbf{D}=\mathbf{U} \Lambda^{1 / 2}$. By applying a decorrelating transformation $\boldsymbol{z}=\mathbf{D} \boldsymbol{w}, z_{i}$ is given by $z_{i}=\sum_{j=1}^{3} d_{i j} w_{j}$, where $d_{i j}$ is the $(i, j)$-th element of D. Substituting into (7), we have

$$
\begin{aligned}
\operatorname{Pr}(\mathrm{A} \cap \mathrm{B}) & =\frac{1}{(\sqrt{\pi})^{3}} \int_{-\infty}^{\infty} \int_{-\infty}^{\infty} \int_{-\infty}^{\infty} \exp \left[\sum_{i=1}^{3} \frac{-\Gamma}{g\left(\sum_{j=1}^{3} d_{i j} w_{j}\right)}\right] \\
& \cdot \exp \left(-w_{1}^{2}-w_{2}^{2}-w_{3}^{2}\right) d w_{1} d w_{2} d w_{3} .
\end{aligned}
$$

By applying the Gaussian-Hermite integration technique to $w_{1}, w_{2}$ and $w_{3}$, we obtain

$$
\begin{aligned}
\operatorname{Pr}(\mathrm{A} \cap \mathrm{B}) & \approx \frac{1}{(\sqrt{\pi})^{3}} \sum_{n_{1}=1}^{N_{p}} \sum_{n_{2}=1}^{N_{p}} \sum_{n_{3}=1}^{N_{p}} H_{n_{1}} H_{n_{2}} H_{n_{3}} \\
& \cdot \exp \left[\sum_{i=1}^{3}-\Gamma 10-\left(\sqrt{2} \sigma_{i}\left(\sum_{j=1}^{3} d_{i j} \theta_{n_{j}}\right)+\mu_{i}\right) / 10\right] .
\end{aligned}
$$

Similarly, for the third term in (5), the normalized correlation matrix $\mathbf{S}$ is given as

$$
\mathbf{S}=\left[\begin{array}{cc}
1 & \rho_{23} \\
\rho_{23} & 1
\end{array}\right] .
$$

Denote the square root of the normalized correlation matrix $\mathbf{S}$ as $\mathbf{F}$, which is also obtained by eigendecomposition. Using the decorrelating transformation $\boldsymbol{z}=\mathbf{F} \boldsymbol{v}, z_{i}$ is given as $z_{i}=\sum_{j=1}^{2} f_{i j} v_{j}$, where $f_{i j}$ is the $(i, j)$-th element of 
F. As before, by applying the Gaussian-Hermite integration technique to $v_{1}$ and $v_{2}$, we can obtain

$$
\begin{aligned}
& \operatorname{Pr}(\mathrm{B}) \approx \frac{1}{\pi} \sum_{n_{1}=1}^{N_{p}} \sum_{n_{2}=1}^{N_{p}} H_{n_{1}} H_{n_{2}} \\
& \cdot \exp \left[\sum_{i=1}^{2}-\Gamma 10-\left(\sqrt{2} \sigma_{i+1}\left(\sum_{j=1}^{2} f_{i j} \theta_{n_{j}}\right)+\mu_{i+1}\right) / 10\right] .
\end{aligned}
$$

Finally, by substituting (6), (10) and (12) into (5), the outage probability for the DF cooperative diversity systems considering the effects of independent Rayleigh fading and correlated shadowing is obtained.

\section{Numerical and simulation results}

In this section, we present some numerical and simulation results to validate our analysis. For simplicity, we assume the source, relay and destination are located at the vertices of an equilateral triangle and therefore the mean SNR of the source-destination, source-relay, and relay-destination links are the same, i.e., $\mu_{1}=\mu_{2}=\mu_{3}=\mu$. The SNR threshold $\Gamma$ is set to $10 \mathrm{~dB}$ in all the simulations.

In Fig. 1, we show the effect of correlations on the outage performance for $\rho_{1}=\rho_{2}=\rho_{3}=\rho, \sigma_{1}=8 \mathrm{~dB}, \sigma_{2}=6 \mathrm{~dB}$ and $\sigma_{3}=4 \mathrm{~dB}$. It is observed from Fig. 1 that, the proposed evaluations agree well with the Monte Carlo simulation results, and the outage performance degrades as the correlation coefficients of shadowing increase.

In Fig. 2, we show the relationship between the standard deviations of shadowing and the outage performance, where $\sigma_{1}=\sigma_{2}=\sigma_{3}=\sigma \mathrm{dB}, \rho_{12}=$

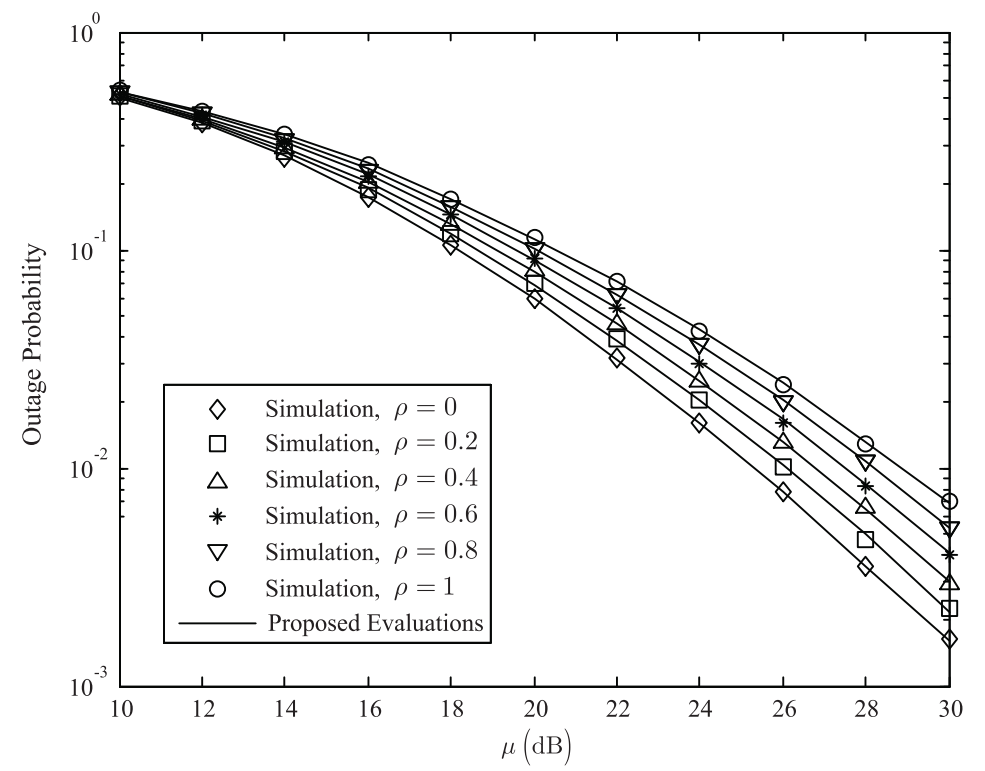

Fig. 1. The effect of correlations on the outage probability, $\rho_{1}=\rho_{2}=\rho_{3}=\rho, \sigma_{1}=8 \mathrm{~dB}, \sigma_{2}=6 \mathrm{~dB}$ and $\sigma_{3}=4 \mathrm{~dB}$. 


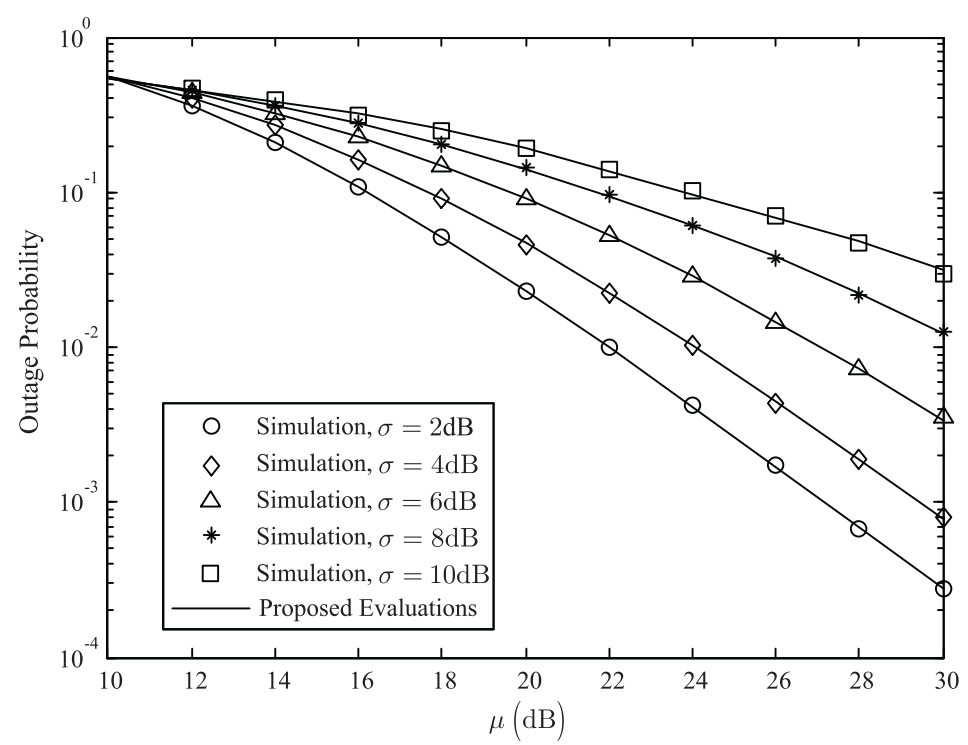

Fig. 2. The effect of standard deviations on the outage probability, $\sigma_{1}=\sigma_{2}=\sigma_{3}=\sigma \mathrm{dB}, \rho_{12}=0.6$, $\rho_{13}=0.8$ and $\rho_{23}=0.2$.

$0.6, \rho_{13}=0.8$ and $\rho_{23}=0.2$. We can observe that the standard deviations of shadowing have a significant impact on the outage performance. It is further proved from Fig. 2 that the proposed evaluations are very accurate.

\section{Conclusion}

In this letter, the outage probability of DF cooperative diversity systems with selection combining at the destination was investigated under correlated lognormal shadowed Rayleigh fading channels. By applying a decorrelating transformation, the outage probability was obtained using Gaussian-Hermite integration technique. The numerical results showed that our performance analysis expressions coincide with simulations.

\section{Acknowledgment}

This work is supported by the National Natural Science Foundation of China (61372097, 61271411, 61271164, U1035002/L05, 61001087, 61101034) and the Fundamental Research Funds for the Central Universities (ZYGX2011YB003). 